\title{
ON A SINGLE-SERVER QUEUE WITH FIXED ACCUMULATION LEVEL, STATE DEPENDENT SERVICE, AND SEMI-MARKOV MODULATED INPUT FLOW
}

\author{
JEWGENI H. DSHALALOW \\ and \\ GARY RUSSELL \\ Department of Applied Mathematics \\ Florida Institute of Technology \\ Melbourne, FL 32901, USA
}

(Received July 29,1991)

\begin{abstract}
The authors study the queueing process in a single-server queueing system with state dependent service and with the input modulated by a semi-Markov process embedded in the queueing process. It is also assumed that the server capacity is $r \geq 1$ and that any service act will not begin until the queue accumulates at least $r$ units. In this model, therefore, idle periods also depend upon the queue length.

The authors establish an ergodicity criterion for the queueing process and evaluate explicitly its stationary distribution and other characteristics of the system, such as the mean service cycle, intensity of the system, intensity of the input stream, distribution of the idle period, and the mean busy period. Various special cases are treated.
\end{abstract}

KEY WORDS AND PHRASES. Queueing process, semi-Markov process, semi-regenerative process, embedded Markov chain, semi-Markov modulated Poisson process, equilibrium, continuous time parameter process, service cycle, idle period, busy period, intensity of the process.

1991 AMS SUBJECT CLASSIFICATION CODES. Primary 60K10, 60K15, 60K25, Secondary 90B22, 90B25.

\section{INTRODUCTION.}

Many standard queueing systems operate on the assumption that input and service parameters are independent of the state of the system; to assume otherwise is frequently regarded as too unwieldy. In this article we propose a class of queueing systems which can be analyzed rather thoroughly even though the input and service parameters are state dependent. We add the provision that the service is delayed until the service batch size reaches the server's capacity. We will show that such a class of systems readily avails itself to the appropriate analytical techniques.

Allowing state dependence makes the model more versatile. Also, if the delay in service seems too restrictive, models that do not make this assumption are already available. 
However, for the class of systems under consideration, we assume that $r(\geq 1)$ units are necessary for service, and if fewer are available the server waits for more units to arrive. The different possible values of $r$, as well as the formation of the idle period (which in our case does not necessarily end with the arrival of the next unit), allows our system to encompass more situations. Applications in transportation seem probable.

Other authors, such as Neuts, Sumita, and Takahashi (see [4]), have studied a Poisson process modulated by a Markov process. In this article we consider the more general case of a Poisson process modulated by a semi-Markov process. We also assume the service process is state dependent. After a formal description we study the embedded queueing process constructed over the sequence of instants of service completion (under no restriction to service time distributions). We extend the result for the continuous time parameter process by using techniques appropriate for semi-regenerative processes. We establish a necessary and sufficient criterion for ergodicity and give explicit formulas for the limiting distributions of the processes. We derive the mean service cycle, intensity of the system, intensity of the input stream, distribution of the idle period, and the mean busy period. Examples are presented throughout the paper.

\section{FORMAL DESCRIPTION OF THE SYSTEM}

Let $\left\{\Omega, \mathcal{F},\left(P^{x}\right)_{x \in E}, Q(t) ; t \geq 0\right\} \rightarrow E=\{0,1, \ldots\}$ denote the number of units in a single-server queueing system at time $t$, let $\left\{T_{n} ; n \in N, T_{0}=0,\right\}$ be the sequence of successive instants of service completion, let $Q_{n}=Q\left(T_{n}+0\right)$, let $C(\cdot)$ be the counting measure associated with the point process $\left\{T_{n}\right\}$, and let $\xi(t)=Q\left(T_{C(t)}+0\right), t \geq 0$. Then the input is a Poisson process modulated by $\{\xi(t)\}$ due to a definition to follow.

Let $\xi(t)$ be an integer-valued jump process (with successive jumps at $T_{n}, n \in N$, noting that 0 is the increment of a jump in the case of $\left.Q_{n-1}=Q_{n}\right)$ and let $\left\{\tau_{k} ; k \in N\right\}$ be a non-stationary orderly Poisson point process with its intensity function $\lambda(t)$. Then we call the doubly stochastic Poisson point process with intensity $\lambda(\xi(t))$ the Poisson process modulated by the jump process $\{\xi(t)\}$ and it is denoted by $\left\{\tau_{n}^{\xi}\right\}$. Let $N^{\xi}(\cdot)$ denote the associated counting measure. [For a formal definition of modulated processes see Dshalalow [3].]

If the queue length $Q_{n}$ is at least $r$, then at time $T_{n}+0$ the server takes a batch of units of size $r$ from the queue (according to the FIFO discipline) and serves it for a random time $\sigma_{n+1}$. Otherwise, the server idles until the queue length $Q(t)$ first reaches level $r$ after $T_{n}$ and then it begins to process a group of $r$ units taken from the waiting room of infinite capacity (again, according to the FIFO discipline) with actual service time again equal to $\sigma_{n+1}$. In both cases we assume that $\sigma_{n+1}$ has a probability distribution function $B_{Q_{n}} \in\left\{B_{0}, B_{1}, \ldots\right\}, B_{i}$ being an arbitrary distribution function with finite mean $b_{i}$.

\section{EMBEDDED PROCESS $\left\{Q_{n}\right\}$.}

Let $V_{n}=N^{\xi}\left(\sigma_{n}\right)$. Then the process $\left\{Q_{n}\right\}$ is defined recursively by

$$
Q_{n+1}=\left(Q_{n}-r\right)^{+}+V_{n+1}
$$

where operator $(f)^{+}$is defined as $(f)^{+}=\sup \{f, 0\}$. From relation (3.1) and the nature of the input process it follows that the process $\left\{\Omega, \mathcal{F},\left(P^{x}\right)_{x \in E}, Q(t) ; t \geq 0\right\} \rightarrow E$ has at $T_{n}, n \geq 1$, the locally strong Markov property (see definition A.3 in Appendix) and that $\left\{\Omega, \mathscr{F},\left(P^{x}\right)_{x \in E}\right.$, 
$\left.Q_{n} ; n \in \mathbb{N}_{0}\right\} \rightarrow E$ is a homogeneous Markov chain with transition probability matrix $T=\left(p_{i}\right)$. Let $A_{\mathrm{a}}(z)$ denote the generating function of $i$ th row of matrix $T$. From $A_{1}(z)=E^{\prime}\left[z^{Q_{1}}\right]$ and (3.1) we obtain

$$
A_{i}(z)=z^{(i-r)+} \beta_{i}\left(\lambda_{1}-\lambda_{i} z\right), i \in E
$$

where $\beta_{i}(\theta), \operatorname{Re}(\theta) \geq 0$, is the Laplace-Stieltjes transform of the probability distribution function $B_{i}$ and $\lambda_{i}=\lambda(i)$. For analytical advantage and with very little sacrifice of generality we drop the modulation and service control when the queue length exceeds a fixed (perhaps very large) level $N$. In other words, we assume that

$$
B_{i}(x)=B(x), \beta_{i}(\theta)=\beta(\theta), b_{i}=b, \lambda_{i}=\lambda, i>N
$$

Without loss of generality we also assume that $N \geq r-1$.

Given assumption (AS), we can show that the transition probability matrix $T$ is reduced to a form of the $\Delta_{r, N}$-matrix introduced and studied in [1]. According to theorem A.1 (see Appendix), the Markov chain $\left\{Q_{n}\right\}$ is recurrent-positive if and only if

and

$$
\lim _{z \rightarrow 1: z \in B(0,1)} \frac{d}{d z} A_{i}(z)<\infty, i=0,1, \ldots, N
$$

$$
\lim _{z \rightarrow 1: z \in B(0,1)} \frac{d}{d z} \beta(\lambda-\lambda z)<r
$$

Condition (3.3) is obviously met and condition (3.4) is equivalent to

$$
\rho=\lambda b<r
$$

Given that $\rho<r$, the Markov chain $\left\{Q_{n}\right\}$ is ergodic. Let $P=\left(p_{x} ; x \in E\right)$ denote the invariant probability measure of operator $T$ and let $P(z)$ be the generating function of vector $P$. Now we formulate the main result of this section.

THEOREM 1. The embedded queueing process $\left\{Q_{n}\right\}$ is ergodic if and only if $\rho<\mathrm{r}$. Under this condition, $P(z)$ satisfies the equation

$$
\Psi\left(p_{0}, \ldots, p_{N}\right)=P(z)=\frac{\sum_{i=0}^{N} p_{i}\left\{z^{r} A_{i}(z)-z^{i} \beta(\lambda-\lambda z)\right\}}{z^{r}-\beta(\lambda-\lambda z)}
$$

with $A_{i}(z)$ determined by (3.2). Probabilities $p_{0}, \ldots, p_{N}$ form the unique solution to the following system of linear equations:

$$
\begin{gathered}
\left.\sum_{i=0}^{N} p_{i} \frac{d^{k}}{d z^{k}}\left\{A_{i}(z)-z^{i}\right\}\right|_{z=z_{s}}=0, k=0, \ldots, k_{s}-1, s=1, \ldots, S \\
\sum_{i=0}^{N} p_{i}\left[\rho_{i}-\rho+(r-i)^{+}\right]=r-\rho
\end{gathered}
$$

where $\rho_{i}=\lambda_{i} b_{i}, z_{s}$ are the roots of $z^{N+1}-z^{N+1-r} \beta(\lambda-\lambda z)$ in the region $\bar{B}(0,1) \backslash\{1\}$ with their multiplicities $\mathrm{k}_{\mathrm{s}}$ such that $\sum_{s=1}^{S} k_{\mathrm{s}}=N$.

PROOF. Formula (3.6a) follows from $P(z)=\sum_{i c E} p_{i} A_{i}(z)$ and (3.2). It is easy to modify formula (3.6a) into

$$
\sum_{i=N+1}^{\infty} p_{i} z^{i-(N+1)}=\frac{\sum_{i=0}^{N} p_{i}\left\{A_{i}(z)-z^{i}\right\}}{z^{N+1}-z^{N+1-r} \beta(\lambda-\lambda z)}
$$

Obviously, $\sum_{i=N+1}^{\infty} p_{i} z^{i-(N+1)}$ is analytic in $B(0,1)$ and continuous on $\partial B(0,1)$. According to theorem A.2, the function $z \mapsto z-\beta(\lambda-\lambda z$ ) must have exactly $r$ zeros (counted with their multiplicities) in $\bar{B}(0,1)$, and all zeros on the boundary $\partial B(0,1)$, including the root 1 , must be simple after we meet the ergodicity condition $\rho<r$. Therefore, the denominator in the right hand side of (3.6d) has exactly $N$ roots in the region $\bar{B}(0,1) \backslash\{1\}$ and this, along with $(P, 1)=1$ (which is equivalent to (3.6c)), yields the equations in (3.6b) and (3.6c).

Now we prove the uniqueness of $\left\{p_{0}, \ldots, p_{N}\right\}$. Suppose that the system of equations 
(3.6b) and (3.6c) has another solution $p^{*}=\left\{p_{i}^{*} ; i=0, \ldots, N\right\}$. We substitute $p^{*}$ into (3.6a) to obtain the generating function $P^{*}(z)$. Then, $P^{*}(z)$ is analytic in $B(0,1)$ and continuous on $\partial B(0,1)$. Therefore, $P^{*}=\left\{p_{i}^{*} ; i \in \Psi\right\} \in\left(l^{1},\|\cdot\|\right)$. Obviously, equations $P^{*}(z)=\sum_{u \in \psi} p_{i}^{*} A_{i}(z)$ and

$$
P^{*}(z)=\frac{\sum_{i=0}^{N} p_{i}^{*}\left\{z A_{i}(z)-z^{i} \beta(\lambda-\lambda z)\right\}}{z^{z}-\beta(\lambda-\lambda z)}
$$

are equivalent. The last equation is also equivalent to $P^{*}=P^{*} A$. Since $p^{*}$ satisfies (3.6c) it follows that $\left(P^{*}, 1\right)=1$. Thus, the system of equations $x=x A,(x, 1)=1$ has two different solutions in $\left(l^{1},\|\cdot\|\right)$ which is impossible.

\section{EXAMPLES AND APPLICATIONS.}

\section{DEFINITIONS 2.}

(i) Let $\beta,=E^{J}\left[T_{1}\right]$, the mean sojourn time of the process $\{\xi(t)\}$ in state $\{j\}$, and let $\beta=(\beta, ; j \in E)^{\mathrm{T}}$. Then $\boldsymbol{P} \boldsymbol{\beta}$ is the mean service cycle of the system, where $\boldsymbol{P}$ denotes the stationary probability distribution vector of the embedded queueing process $\left\{Q_{n}\right\}$.

(ii) Let $\lambda=\left(\lambda_{x} ; x \in E\right)^{\mathrm{T}}$ and let $\rho=\beta * \lambda$ be the Hadamard (entry-wise) product of vectors $\beta$ and $\lambda$. We call the scalar product $\boldsymbol{P} \rho$ the intensity of the system.

The concept of "intensity of the system" goes back to the classical $M / G / 1$ system, where $P \rho$ reduces to $\rho=\lambda b$. It is worth noting that the intensity of the system and the capacity of the server (in our case $r$ ) coincide, as stated in proposition 4 and proved thereafter.

PROPOSITION 3. Given the equilibrium condition $\rho<r$, the mean service cycle satisfies the following equation:

$$
P \beta=b+\sum_{j=0}^{N} p_{j}\left[(b,-b)+\frac{1}{\lambda_{j}}(r-i)^{+}\right]
$$

PROOF. Obviously, $\beta_{j}=b_{j}+(r-j)^{+} / \lambda_{\text {, }}$. The statement is now due to elementary algebraic transformations.

PROPOSITION 4. Given the ergodicity condition $\rho<r$, the intensity of the system and and the capacity of the server coincide.

PROOF. From definition of $\boldsymbol{P} \rho$ and considerations as in proposition 3 it follows that

$$
P \rho=\rho+\sum_{j=0}^{N} p_{j}\left[\left(\rho_{j}-\rho\right)+(r-i)^{+}\right]
$$

The statement is due to relation (3.6c) and elementary algebraic transformations.

\section{EXAMPLES 5.}

(i) Consider a special case of our model with $r=2, N=4$ and with $B$ as a negative exponential distribution with parameter $\frac{1}{b}$. However, we retain all other assumptions about the modulation and service control having $B_{0}, \ldots, B_{4}$ arbitrary. For this case we obtain $\beta(\lambda-\lambda z)$ $=(1+\rho-\rho z)^{-1}$ and it follows that the only root of the equation $z^{2}-\beta(\lambda-\lambda z)$ inside the ball $B(0,1)$ is $z_{1}=\frac{1-\sqrt{1+4 \rho}}{2 \rho}$. Thus for equation (3.6b) we will be using $z_{1}$ with multiplicity one and 0 with multiplicity three. This will give 4 of total 5 equations in the unknowns $p_{0}, \ldots, p_{4}$ :

$$
\begin{gathered}
\sum_{i=0}^{4}\left\{z_{1}^{(i-2)^{+}} \beta_{i}\left(\lambda_{i}-\lambda_{i} z\right)-z_{1}^{i}\right\} p_{i}=0 \\
\lambda_{0}^{2} \beta_{0}^{\prime \prime}\left(\lambda_{0}\right) p_{0}+\lambda_{1}^{2} \beta_{1}^{\prime \prime}\left(\lambda_{1}\right) p_{1}+\left[\lambda_{2}^{2} \beta_{2}^{\prime \prime}\left(\lambda_{2}\right)-2\right] p_{2}-2 \lambda_{3} \beta_{3}^{\prime}\left(\lambda_{3}\right)+2 \beta_{4}\left(\lambda_{4}\right) p_{4}=0 \\
-\lambda_{0} \beta_{0}^{\prime}\left(\lambda_{0}\right) p_{0}-\left[\lambda_{1} \beta_{1}^{\prime}\left(\lambda_{1}\right)+1\right] p_{1}-\lambda_{2} \beta_{2}^{\prime}\left(\lambda_{2}\right) p_{2}+\beta_{3}\left(\lambda_{3}\right) p_{3}=0 \\
{\left[\beta_{0}\left(\lambda_{0}\right)-1\right] p_{0}+\beta_{1}\left(\lambda_{1}\right) p_{1}+\beta_{2}\left(\lambda_{2}\right) p_{2}=0 .}
\end{gathered}
$$


The fifth equation will be (3.6c) with $r=2$ and $N=4$. This system can be solved by elementary algebraic methods. The solution of this system will then be put into equation (3.6a) to have the generating function $P(z)$ in an explicit form.

(ii) By dropping the modulation and service control and setting $r=1$, we immediately arrive at the classical formula by Kendall established for the model $M / G / 1$.

\section{CONTINUOUS TIME PARAMETER PROCESS $\{Q(t)\}$.}

In this section our main objective will be the derivation of the stationary distribution of the queueing process with continuous time parameter. Prior to this, we will be concerned with some preliminaries.

From section 3 and definition A.4, it follows that $\left\{\Omega, \mathscr{F},\left(P^{x}\right)_{x \in E}, Q(t) ; t \geq 0\right\} \rightarrow(E$, $\mathscr{B}(E)$ ) is a semi-regenerative process with conditional regenerations at points $T_{n}, n \in \mathbb{N}$. Let $\left\{\Omega, \mathcal{F},\left(P^{x}\right)_{x \in E}, Q_{n}, T_{n}: n=0,1, \ldots\right\} \rightarrow\left(E \times \mathbf{R}_{+}, \mathscr{B}\left(E \times \mathbb{R}_{+}\right)\right)$be the associated Markov renewal process and let $\varphi(t)$ be the corresponding semi-Markov kernel. With a very mild restriction to the probability distribution functions $B_{i}$, we can specify that the elements of $\varphi(t)$ are not step functions and thus $\left\{Q_{n}, T_{n}\right\}$ is aperiodic. By proposition 3, the mean service cycle $P \beta$, which is also the mean inter-renewal time of the Markov renewal process, is obviously finite. Therefore, following definition A.5 and given that $\rho<r$, the Markov renewal process is ergodic.

It also follows that the jump process $\left\{\Omega, \mathcal{F},\left(P^{x}\right)_{x \in E}, \xi(t) ; t \geq 0\right\} \rightarrow E$, defined in section 2 , is the minimal semi-Markov process associated with the Markov renewal process $\left\{Q_{n}, T_{n}\right\}$ and therefore, following the definition in section 2, the input process $\left\{\Omega, \mathcal{F},\left(P^{x}\right)_{x \in E}, N^{\xi}\right\} \rightarrow E$ is a Poisson process modulated by the semi-Markov process $\{\xi(t)\}$.

Let $K(t)=\left(K_{j k}(t) ; j, k \in E\right)$ be the semi-regenerative kernel (see definition A.6). The following statement holds true.

LEMMA 6. The semi-regenerative kernel satisfies the following equations:

$$
K_{j k}(t)=\left\{\begin{array}{cc}
\hat{\pi}_{\lambda_{j} t}(k-j), & 0 \leq j \leq k<r \\
\int_{0}^{t} \varepsilon\left(\lambda_{j}, r-j, t-u\right) \hat{\pi}_{\lambda_{j} u}(k-r)\left(1-B_{j}(u)\right) d u, & 0 \leq j<r \leq k \\
\hat{\pi}_{\lambda_{j} t}(k-j)\left[1-B_{j}(t)\right], & r \leq j \leq k \\
0, & 0 \leq k<j,
\end{array}\right.
$$

with $\left(\hat{\pi}_{y} ; y \in \mathbf{R}_{+}\right)$the Poisson semi-group and $\varepsilon(\lambda, n, \cdot)$ the Erlang- $n$ probability density function with parameter $\lambda$.

PROOF. The above assertion follows from straightforward probability arguments.

Now we are ready to apply the Main Convergence Theorem to the semi-regenerative kernel in the form of corollary A.8, thereby arriving at the stationary distribution of the queueing process $\{Q(t)\}$.

THEOREM 7. Given the equilibrium condition $\rho<r$ for the embedded process $\left\{Q_{n}\right\}$, the stationary distribution $\pi=\left(\pi_{x} ; x \in E\right)$ of the queueing process $\{Q(t)\}$ exists; it is independent of any initial distribution and is expressed in terms of the generating function $\pi(z)$ of $\pi$ by the following formula:

$$
\begin{array}{r}
P \beta \pi(z)(1-z)=\frac{1}{\lambda}[1-\beta(\lambda-\lambda z)] P(z)+\sum_{j=0}^{N} p_{j} z^{j}\left(\frac{1}{\lambda_{j}}-\frac{1}{\lambda}\right) \\
-\sum_{j=0}^{N} p_{j}\left[\frac{1}{\lambda_{j}} z^{r} A_{j}(z)-\frac{1}{\lambda} z^{j} \beta(\lambda-\lambda z)\right]
\end{array}
$$


where $P(z)$ is the generating function of $\boldsymbol{P}, \boldsymbol{P} \boldsymbol{\beta}$ is determined in proposition 3 , and $A_{j}(z)$ is defined in (3.2).

PROOF. Recall that the Markov renewal process $\left\{Q_{n}, T_{n}\right\}$ is ergodic if $\rho<r$. By corollary A.8 the semi-regenerative process $\{Q(t)\}$ has a unique stationary distribution $\pi$ provided that $\rho<r$. We can see that the semi-regenerative kernel is Riemann integrable over $\mathbf{R}_{+}$. Thus, following corollary A.8, we need to find the integrated semi-regenerative kernel $H$ (which is done with routine calculus) and then the generating function $h,(z)$ for each row of $H$. First we find that

Then,

$$
\sum_{p=j}^{\infty} z^{p} \int_{0}^{\infty} \hat{\pi}_{\lambda, u}(p-j)\left[1-B_{j}(u)\right] d u=z^{j} \frac{1-\beta_{j}\left(\lambda_{j}-\lambda_{j} z\right)}{\lambda_{j}(1-z)}=h_{j}(z), j \geq r
$$

$$
h_{j}(z)=z^{j} \frac{1-z^{-j} \beta_{j}\left(\lambda_{j}-\lambda_{j} z\right)}{\lambda_{j}(1-z)}, 0 \leq j<r
$$

Formula (5.2a) now follows from corollary A.8, equations (5.2b) and (5.2c) and some algebraic transformations.

\section{EXAMPLES 8.}

(i) By dropping the modulation of the input process we obtain from proposition 4 that $P \beta=\frac{r}{\lambda}$ and $\pi(z)=\frac{1-z^{r}}{r(1-z)} P(z)$.

(ii) By using obvious probability arguments we derive the probability density function of

an idle period in the steady state:

The mean idle period $g$ in the steady state is then

$$
u \mapsto \frac{\sum_{i=0}^{r-1} p_{i} \varepsilon\left(\lambda_{i}, r-i, u\right)}{\sum_{i=0}^{r-1} p_{i}} .
$$

$$
g=\frac{\sum_{i=0}^{r-1} p_{i}(r-i) \frac{1}{\lambda_{i}}}{\sum_{i=0}^{r-1} p_{i}}
$$

(iii) Formula (5.3a) and theorem 7 allow us to derive the mean busy period $B$ in equilibrium. Clearly $\sum_{i=0}^{r-1} \pi_{i}$ is the probability that the server idles. On the other hand, it also equals $\frac{g}{g+B}$. Thus we have

$$
\mathfrak{B}=\frac{g \sum_{i=r}^{\infty} \pi_{i}}{\sum_{i=0}^{r-1} \pi_{i}}
$$

(iv) Now we turn to the special case in example 5 (i) applying its results for the process with continuous time parameter. We use probabilities $p_{0}, \ldots, p_{4}$, substituting them into formulas (5.2a) and (4.1) for $r=2$ and $N=4$, thereby reducing the generating function $\pi(z)$ to an explicit form.

(v) If the input is a stationary Poisson process then its intensity is $\lambda$, which is also the mean number of arrivals per unit time. In the case of a modulated input process its intensity is no longer a trivial fact. We define the intensity of any counting measure $N$ by the formula

$$
\kappa=\lim _{t \rightarrow \infty} \frac{1}{t} \mu_{t}(x),
$$

where $\mu_{t}(x)=E^{x}[N([0, t])]$. We will apply ergodic theorem 3.9 established in Dshalalow [3] for more general Poisson process modulated by a semi-Markov process:

$$
\kappa=P \rho / P \beta \text {, }
$$

where by proposition 4.3 $P \beta=r$ and $P \beta$ satisfies equation (4.1) and thus we have:

$$
\kappa=\frac{r}{\boldsymbol{P} \boldsymbol{\beta}}
$$

A trivial special case appears when we drop the modulation of the input and therefore use 
formula (4.1) combining it with formula (5.3b). Then $\kappa=\lambda$, as it should be.

Another interesting special case is due to the assumption that $b_{i}=b$ and $r=1$ (observe that we only require that the service means $b_{0}, b_{1}, \ldots$ are equal but do not restrict the corresponding distributions). Then we have that $\kappa$ equals the reciprocal of $P \beta=b+p_{0} \frac{1}{\lambda_{0}}$.

\section{APPENDIX}

THEOREM A.1 (Abolnikov and Dukhovny [1]). Let $\left\{Q_{n}\right\}$ be an irreducible aperiodic Markov chain with the transition probability matrix $A$ in the form of a $\Delta_{r, N^{-m a t r i x .} \text { Then }}$ $\left\{Q_{n}\right\}$ is recurrent-positive if and only if

and

$$
\lim _{z \rightarrow 1: z \in B(0,1)} \frac{d}{d z} A_{i}(z)<\infty, i=0,1, \ldots, N
$$

$$
\lim _{z \rightarrow 1: z \in B(0,1)} \frac{d}{d z} \beta(\lambda-\lambda z)<r
$$

THEOREM A.2 (Abolnikov and Dukhovny [1]). Under the condition of (A.1b) the function $z^{5}-\beta(\lambda-\lambda z)$ has exactly $\mathrm{r}$ roots belonging to the closed unit ball $\bar{B}(0,1)=\{z \in \mathrm{C}$ : $\|z\| \leq 1\}$. Those roots lying on the boundary $\partial B(0,1)$ are simple.

DEFINITION A.3. Let $T$ be a stopping time for a stochastic process $\left\{\Omega, \mathcal{F},\left(P^{x}\right)_{x \in E}\right.$, $Z(t) ; t \geq 0\} \rightarrow(E, \mathfrak{B}(E))$. The process $\{Z(t)\}$ is said to have the locally strong Markov property at $T$ if for each bounded random variable $\zeta: \Omega \rightarrow E^{r}$ and for each Baire function $f$ : $E^{r} \rightarrow \boldsymbol{R}, r=1,2, \ldots$, it holds true that

$$
E^{x}\left[f \circ \zeta \circ \theta_{T} \mid \mathcal{F}_{T}\right]=E^{Z_{T}}[f \circ \zeta] P^{x} \text {-a.s. on }\{T<\infty\},
$$

where $\theta_{y}$ is the shift operator.

DEFINITION A.4. A stochastic process $\left\{\Omega, \mathscr{F},\left(P^{x}\right)_{x \in E}, Z(t) ; t \geq 0\right\} \rightarrow(E, \mathfrak{B}(E))$ with $E \preceq N$ is called semi-regenerative if

a) there is a point process $\left\{T_{n}\right\}$ on $\boldsymbol{R}_{+}$such that $T_{n} \rightarrow \infty(n \rightarrow \infty)$ and such that each $T_{n}$ is a stopping time relative to the canonic filtering $\sigma\left(Z_{y} ; y \leq t\right)$,

b) the process $\{Z(t)\}$ has the locally strong Markov property at $T_{n}, n=1,2, \ldots$, and

c) $\left\{Z\left(T_{n}+0\right), T_{n} ; n=0,1, \ldots\right\}$ is a Markov renewal process.

DEFINITION A.5. Let $\left\{X_{n}, T_{n}\right\}$ be an irreducible aperiodic Markov renewal process with discrete state space $E$. Let $\beta_{x}=E^{x}\left[T_{1}\right]$ be the mean sojourn time of the Markov renewal process in state $\{x\}$ and let $\beta=\left(\beta_{x} ; x \in E\right)^{\mathrm{T}}$. Suppose that the embedded Markov chain $\left\{X_{n}\right\}$ is ergodic with stationary distribution $P$. We call $P \beta$ the mean inter-renewal time. We call the Markov renewal process recurrent-positive if its mean inter-renewal time is finite. An irreducible aperiodic and recurrent-positive Markov renewal process is called ergodic.

DEFINITION A.6. Let $\left\{\Omega, \mathcal{F},\left(P^{x}\right)_{x \in E}, Z(t) ; t \geq 0\right\} \rightarrow(E, \mathfrak{B}(E))$ be a semi-regenerative process relative to the sequence $\left\{T_{n}\right\}$ of stopping times and let

$$
K_{j k}(t)=P^{\prime}\left\{Z(t)=k, T_{1}>t\right\}, j, k \in E .
$$

We will call the functional matrix $K(t)=\left(K_{j k}(t) ; j, k \in E\right)$ the semi-regenerative kernel.

THEOREM A.7 (The Main Convergence Theorem, cf. Çinlar [2], p. 347). Let $\left\{\Omega, \mathcal{F},\left(P^{x}\right)_{x \in E}, Z(t) ; t \geq 0\right\} \rightarrow(E, \mathscr{B}(E))$ be a semi-regenerative stochastic process relative to the sequence $\left\{T_{n}\right\}$ of stopping times and let $K(t)$ be the corresponding semi-regenerative kernel. Suppose that the associated Markov renewal process is ergodic and that the semi-regenerative kernel is Riemann integrable over $\mathbf{R}_{+}$. Then the stationary distribution $\pi=\left(\pi_{x} ; x \in E\right)$ of the 
process $\{Z(t)\}$ exists and it is determined from the formula:

$$
\pi_{k}=\frac{1}{P \beta} \sum_{\jmath \in E} p, \int_{0}^{\infty} K_{\jmath k}(t) d t, k \in E
$$

COROLLARY A.8. Let $H=\left(h_{j k} ; j, k \in E\right)=\int_{0}^{\infty} K(t) d t$ (the integrated semi-regenerative kernel), let $h_{j}(z)$ be the generating function of $\mathrm{jth}$ row of matrix $H$, and let $\pi(z)$ be the generating function of vector $\pi$. Then

$$
\pi(z)=\frac{1}{P \beta} \sum_{j \in E} p_{j} h_{j}(z)
$$

PROOF. From (A.7a) we get an equivalent formula in matrix form, $\pi=\frac{P H}{P \beta}$. Finally, formula (A.8a) is the result of elementary algebraic transformations.

\section{REFERENCES}

[1]. ABOLNIKOV, L. and DUKHOVNY, A., Markov chains with transition delta-matrix: ergodicity conditions, invariant probability measures and applications, Journ. Appl. Math. Stoch. Anal., 4, No. 4, 335-355, 1991.

[2]. ÇINLAR, E., Introduction to Stochastic Processes, Prentice Hall, Englewood Cliffs, N.J., 1975.

[3]. DSHALALOW, J., On modulated random measures. Journ. Appl. Math. Stoch. Anal., 4, No.4, 305-312, 1991.

[4]. NEUTS, M. F., SUMITA, U., and TAKAHASHI, Y., Renewal characterization of Markov modulated Poisson processes, Journ. Appl. Math. Simul., 2, No. 1, 53-70, 1989. 


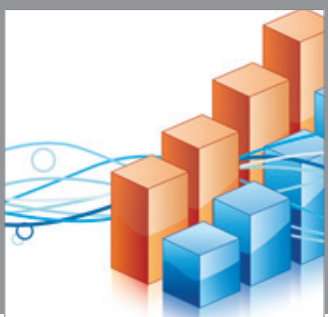

Advances in

Operations Research

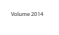

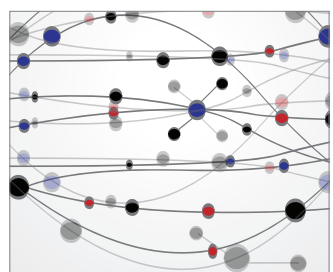

\section{The Scientific} World Journal
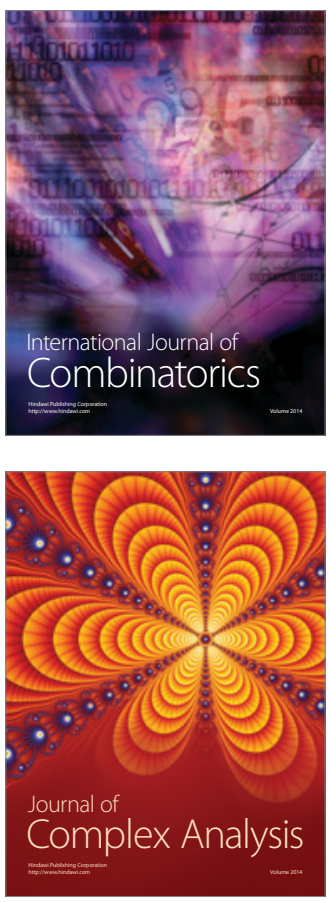

International Journal of

Mathematics and

Mathematical

Sciences
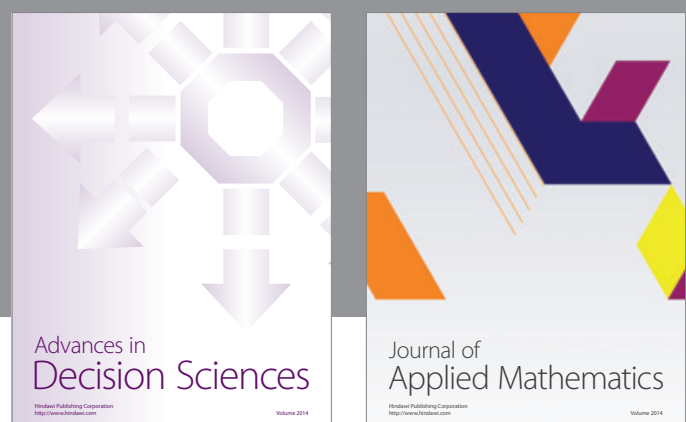

Journal of

Applied Mathematics
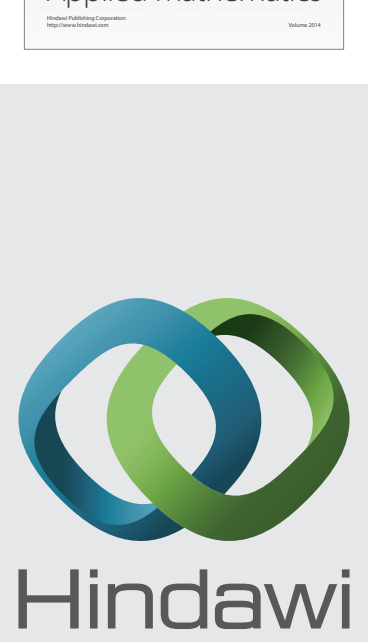

Submit your manuscripts at http://www.hindawi.com
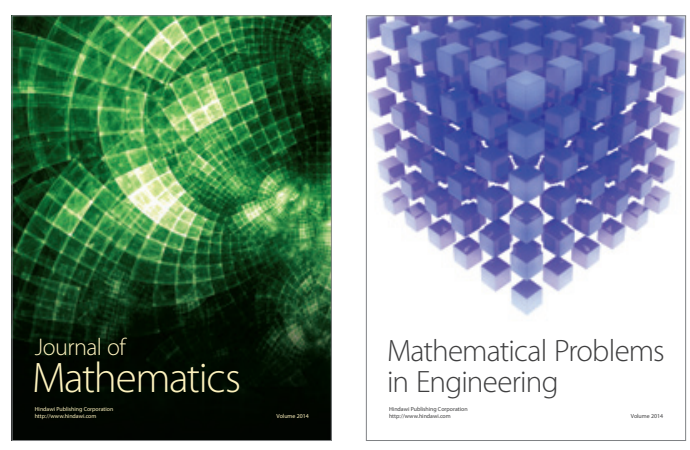

Mathematical Problems in Engineering
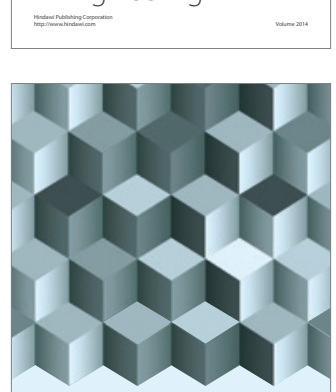

Journal of

Function Spaces
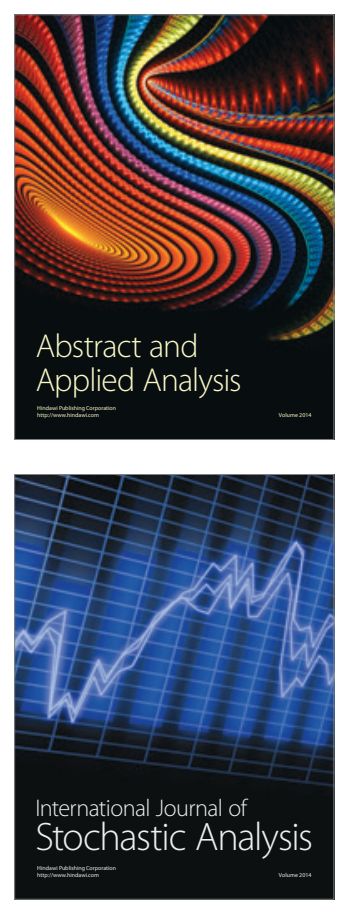

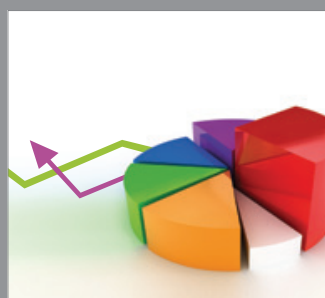

ournal of

Probability and Statistics

Promensencen
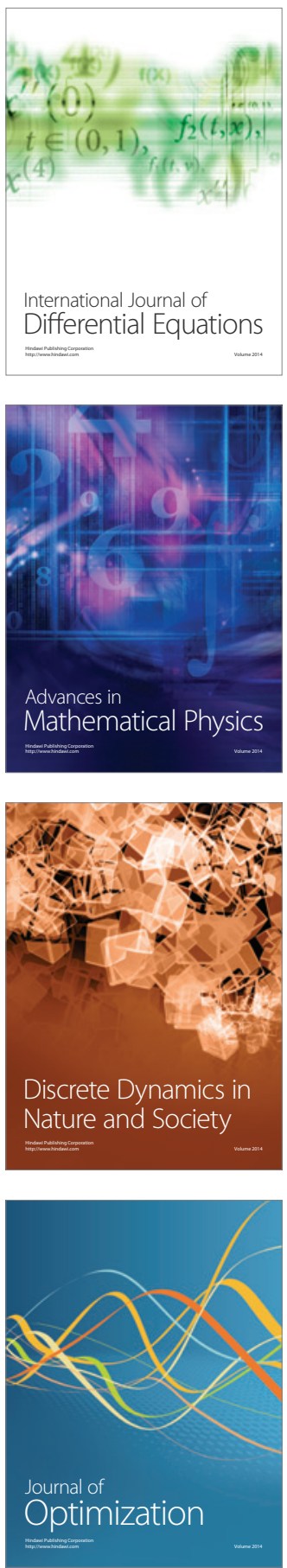